J. Appl. Numer. Optim. 1 (2019), No. 3, pp. 267-276

Available online at http://jano.biemdas.com

https://doi.org/10.23952/jano.1.2019.3.06

\title{
SEMICONTINUITY OF THE COMPOSITION OF SET-VALUED MAP AND SCALARIZATION FUNCTION FOR SETS
}

\author{
KOICHIRO IKE, MENGXUE LIU, YUTO OGATA, TAMAKI TANAKA* \\ Graduate School of Science and Technology, Niigata University, Japan
}

\begin{abstract}
In this paper, we deal with the inheritation of the semicontinuity of set-valued maps via general scalarization for sets, which is regarded as the framework of generalizations of results by Kuwano, Tanaka, and Yamada in 2010. Since the unified scalarization functions for sets satisfy certain desired semicontinuity, our main theorems can be reduced to the results in earlier study.
\end{abstract}

Keywords. Set optimization; Sublinear scalarization; Set relation; Set-valued map; Semicontinuity.

2010 Mathematics Subject Classification. 90C29, 46N10, 49J53, 54C60.

\section{INTRODUCTION}

Continuity is one of the most beneficial concepts in mathematical analysis. For example, it is well known that every continuous real-valued function on a compact set always has a minimum and maximum. Consequently, we can make reasonable models for several social systems and propose approximate solutions of optimization problems by iterative method. For set-valued maps, there are many different kinds of notions on semicontinuity and important results which are not only in theoretical studies but also in many algorithmic analyses related to mathematical programming problems. Accordingly there are many interesting researches in set-valued analysis and various continuity notions for set-valued maps and their properties (see, e.g., $[2,7])$.

On the other hand, scalarization plays a key role in vector optimization and set optimization because optimization problems with set-valued functions become easy to handle by converting vectors or sets into real numbers. Based on Tammer's sublinear scalarization function for vectors $[1,2]$, some authors $([3,6,8,14,15])$ investigated scalarization functions for sets related to set relations [9]. Especially, Nishizawa, Tanaka, and Georgiev [13] suggested interesting results on inherited properties of set-valued maps. Besides, Hamel and Löhne [4] introduced several useful nonlinear scalarization functions for sets with respect to set relations. It provided some motivation for Kuwano, Tanaka, and Yamada to reframe those researches into unification of nonlinear scalarization. In [11], they discussed certain inherited properties on continuity of set-valued maps via the unified scalarizations introduced in [10]. Furthermore, Sonda, Kuwano, and Tanaka [16] extended that work to the case of cone-continuity.

\footnotetext{
${ }^{*}$ Corresponding author.

E-mail addresses: k-ike@m.sc.niigata-u.ac.jp (K. Ike), liumengxue2017@yahoo.co.jp (M. Liu), y-ogata@m.sc. niigata-u.ac.jp (Y. Ogata), tamaki@math.sc.niigata-u.ac.jp (T. Tanaka).

Received May 10, 2019; Accepted September 2, 2019.
}

(C)2019 Journal of Applied and Numerical Optimization 
The aim of this paper is to show certain results on the inheritation of the semicontinuity for set-valued maps via general scalarization for sets, which are regarded as generalizations of results in [11]. In fact, since the unified scalarization functions introduced in [10] satisfy certain desired semicontinuity, our main theorems can be reduced into special cases which are results in [11].

The organization of the paper is as follows. In Section 2, we recall some basic concepts on set relations in set optimization and the scalarization scheme for sets in a real vector space such that each scalarization function has order-monotone property for set relation. In Section 3 , we introduce a new concept of the invariant property for set-valued maps with respect to a binary relationship on a family of sets, which is regarded as some kind of continuity from the view point of order-monotonicity. Then, we show certain inheritation of the semicontinuity of set-valued maps via general scalarization for sets. The last section, Section 4, ends this paper.

\section{SET RELATIONS AND SCALARIZATION FUNCTIONS FOR SETS}

Throughout the paper, let $Y$ be a real topological vector space unless otherwise specified. Let $\theta_{Y}$ be the zero vector in $Y$ and $\mathscr{P}(Y)$ denote the set of all nonempty subsets of $Y$. The topological interior, topological closure, convex hull, and complement of a set $A \in \mathscr{P}(Y)$ are denoted by $\operatorname{int} A, \operatorname{cl} A, \operatorname{co} A$, and $A^{\mathrm{c}}$, respectively.

For given $A, B \in \mathscr{P}(Y)$ and $t \in \mathbb{R}$, the algebraic sum $A+B$ and the scalar multiplication $t A$ are defined as follows:

$$
A+B:=\{a+b \mid a \in A, b \in B\}, \quad t A:=\{t a \mid a \in A\} .
$$

In particular, we denote $A+\{y\}$ by $A+y$ (or $y+A)$ and $(-1) A$ by $-A$ for $A \in \mathscr{P}(Y)$ and $y \in Y$. We define $T y:=\{t y \mid t \in T\}$ for $y \in Y$ and $T \subset \mathbb{R}$.

Let $X$ be a nonempty set and $\preccurlyeq$ a binary relation on $X$. The relation $\preccurlyeq$ is said to be

(1) reflexive if for all $x \in X, x \preccurlyeq x$;

(2) irreflexive if for all $x \in X, x \npreceq x$;

(3) transitive if for all $x, y, z \in X, x \preccurlyeq y$ and $y \preccurlyeq z$ imply $x \preccurlyeq z$;

(4) antisymmetric if for all $x, y \in X, x \preccurlyeq y$ and $y \preccurlyeq x$ imply $x=y$;

(5) complete if for all $x, y \in X, x \preccurlyeq y$ or $y \preccurlyeq x$.

The relation $\preccurlyeq$ is called

(1) a preorder if it is reflexive and transitive;

(2) a strict order if it is irreflexive and transitive;

(3) a partial order if it is reflexive, transitive, and antisymmetric;

(4) a total order if it is reflexive, transitive, antisymmetric, and complete.

A set $C \in \mathscr{P}(Y)$ is called a cone if $t y \in C$ for every $y \in C$ and $t>0$. Let us remark that a cone considered in this paper does not necessarily contain the zero vector $\theta_{Y}$. Let $C$ be a convex cone in $Y$. Then, $C+C=C$ holds, and int $C$ and $\operatorname{cl} C$ are also convex cones. If $\theta_{Y} \in C$, we define a preoder $\leq_{C}$ on $Y$ induced by $C$ as follows:

$$
\text { for } y_{1}, y_{2} \in Y, y_{1} \leq_{C} y_{2} \stackrel{\text { def }}{\Longleftrightarrow} y_{2}-y_{1} \in C \text {. }
$$

This preorder is compatible with the linear structure:

$$
\text { for all } y_{1}, y_{2}, y_{3} \in Y, \quad y_{1} \leq_{C} y_{2} \Longrightarrow y_{1}+y_{3} \leq_{C} y_{2}+y_{3} \text {; }
$$




$$
\text { for all } y_{1}, y_{2} \in Y \text { and } t>0, \quad y_{1} \leq_{C} y_{2} \Longrightarrow t y_{1} \leq_{C} t y_{2} .
$$

If $\theta_{Y} \notin C$, then $\leq_{C}$ is irreflexive and hence a strict order. In addition, assuming that $C$ is pointed (i.e., $C \cap(-C)=\left\{\theta_{Y}\right\}$ ), one can check that $\leq_{C}$ is antisymmetric and becomes a partial order.

Proposition 2.1 ([5]). Let $C$, $C^{\prime}$ be convex cones in $Y$ and $d \in Y$. Assume that $C+(0,+\infty) d \subset C^{\prime}$. Then, for any $y_{1}, y_{2} \in Y$ and $t, t^{\prime} \in \mathbb{R}$ with $t>t^{\prime}$,

$$
y_{1}+t d \leq_{C} y_{2} \Longrightarrow y_{1}+t^{\prime} d \leq_{C^{\prime}} y_{2} .
$$

As generalizations of partial orderings for vectors, we give a definition of certain binary relations between sets in $Y$, called set relations.

Definition 2.1 (set relations, [9]). Let $C$ be a convex cone in $Y$. The six types of set relations are defined by

(1) $A \leq_{C}^{(1)} B \stackrel{\text { def }}{\Longleftrightarrow} \forall a \in A, \forall b \in B, a \leq_{C} b \Longleftrightarrow A \subset \bigcap_{b \in B}(b-C)$;

(2) $A \leq_{C}^{(2 L)} B \stackrel{\text { def }}{\Longleftrightarrow} \exists a \in A$ s.t. $\forall b \in B, a \leq_{C} b \Longleftrightarrow A \cap\left(\bigcap_{b \in B}(b-C)\right) \neq \emptyset$;

(3) $A \leq_{C}^{(3 L)} B \stackrel{\text { def }}{\Longleftrightarrow} \forall b \in B, \exists a \in A$ s.t. $a \leq_{C} b \Longleftrightarrow B \subset A+C$;

(4) $A \leq_{C}^{(2 U)} B \stackrel{\text { def }}{\Longleftrightarrow} \exists b \in B$ s.t. $\forall a \in A, a \leq_{C} b \Longleftrightarrow\left(\bigcap_{a \in A}(a+C)\right) \cap B \neq \emptyset$;

(5) $A \leq_{C}^{(3 U)} B \stackrel{\text { def }}{\Longleftrightarrow} \forall a \in A, \exists b \in B$ s.t. $a \leq_{C} b \Longleftrightarrow A \subset B-C$;

(6) $A \leq_{C}^{(4)} B \stackrel{\text { def }}{\Longleftrightarrow} \exists a \in A, \exists b \in B$ s.t. $a \leq_{C} b \Longleftrightarrow A \cap(B-C) \neq \emptyset$

for $A, B \in \mathscr{P}(Y)$.

Here, the letters $L$ and $U$ stand for "lower" and "upper," respectively.

In general, the relation $\leq_{C}^{(j)}$ is transitive for $j=1,2 L, 2 U, 3 L, 3 U$ and not transitive for $j=4$. If $\theta_{Y} \in C, \leq_{C}^{(j)}$ is reflexive for $j=3 L, 3 U, 4$ and hence a preorder for $j=3 L, 3 U$. If $\theta_{Y} \notin C$, $\leq_{C}^{(j)}$ is irreflexive and hence a strict order for $j=1,2 L, 2 U$. For each $j=1,2 L, 2 U, 3 L, 3 U, 4$, the relation $\leq_{C}^{(j)}$ satisfies the following properties for all $A, B \in \mathscr{P}(Y)$ :

(1) $A \leq_{C}^{(j)} B \Longrightarrow A+y \leq_{C}^{(j)} B+y$ for $y \in Y$;

(2) $A \leq_{C}^{(j)} B \Longrightarrow t A \leq_{C}^{(j)} t B$ for $t>0$.

From the definition, we easily obtain the following implications:

$$
\left\{\begin{array}{l}
A \leq_{C}^{(1)} B \Longrightarrow A \leq_{C}^{(2 L)} B \Longrightarrow A \leq_{C}^{(3 L)} B \Longrightarrow A \leq_{C}^{(4)} B ; \\
A \leq_{C}^{(1)} B \Longrightarrow A \leq_{C}^{(2 U)} B \Longrightarrow A \leq_{C}^{(3 U)} B \Longrightarrow A \leq_{C}^{(4)} B
\end{array}\right.
$$

for $A, B \in \mathscr{P}(Y)$.

Also, we have the following equivalences.

Proposition 2.2. For $A, B \in \mathscr{P}(Y)$, the following statements hold:

(1) $A \leq_{C}^{(1)} B \Longleftrightarrow-B \leq_{C}^{(1)}-A$;

(2) $A \leq_{C}^{(2 L)} B \Longleftrightarrow-B \leq_{C}^{(2 U)}-A$;

(3) $A \leq_{C}^{(3 L)} B \Longleftrightarrow-B \leq_{C}^{(3 U)}-A$;

(4) $A \leq_{C}^{(2 U)} B \Longleftrightarrow-B \leq_{C}^{(2 L)}-A$;

(5) $A \leq_{C}^{(3 U)} B \Longleftrightarrow-B \leq_{C}^{(3 L)}-A$; 
(6) $A \leq_{C}^{(4)} B \Longleftrightarrow-B \leq_{C}^{(4)}-A$.

Proof. The conclusion follows immediately from Definition 2.1.

Proposition 2.3. Let $C, C^{\prime}$ be convex cones in $Y$ and $d \in Y$. Assume that $C+(0,+\infty) d \subset C^{\prime}$. Then, for each $j=1,2 L, 2 U, 3 L, 3 U, 4$, any $A, B \in \mathscr{P}(Y), s, s^{\prime} \in \mathbb{R}$ with $s<s^{\prime}$ and $t, t^{\prime} \in \mathbb{R}$ with $t>t^{\prime}$,

$$
A \leq_{C}^{(j)} B+s d \Longrightarrow A \leq_{C^{\prime}}^{(j)} B+s^{\prime} d
$$

and

$$
A+t d \leq_{C}^{(j)} B \Longrightarrow A+t^{\prime} d \leq_{C^{\prime}}^{(j)} B
$$

Proof. The conclusion follows immediately from Definition 2.1 and Proposition 2.1.

Based on the set relations, we introduce the following scalarization functions for sets in $Y$, which are certain generalization as unification of several nonlinear scalarizations proposed in [13].

Definition 2.2 ([5, 10]). For each $j=1,2 L, 2 U, 3 L, 3 U, 4$, we define

$$
\begin{aligned}
& I_{C}^{(j)}(A ; V, d):=\inf \left\{t \in \mathbb{R} \mid A \leq_{C}^{(j)}(V+t d)\right\}, \\
& S_{C}^{(j)}(A ; V, d):=\sup \left\{t \in \mathbb{R} \mid(V+t d) \leq_{C}^{(j)} A\right\}
\end{aligned}
$$

for $A, V \in \mathscr{P}(Y)$ and $d \in Y ; V$ and $d$ are index parameters for scalarization and play key roles as a reference set and a direction, respectively, as one kind of sublinear-like scalarization for a given set $A$.

Proposition 2.4. Let $A, V \in \mathscr{P}(Y)$ and $d \in Y$. Then, the following statements hold:

$$
\begin{aligned}
-I_{C}^{(1)}(-A ;-V, d) & =S_{C}^{(1)}(A ; V, d) ; \\
-I_{C}^{(2 L)}(-A ;-V, d) & =S_{C}^{(2 U)}(A ; V, d) ; \\
-I_{C}^{(3 L)}(-A ;-V, d) & =S_{C}^{(3 U)}(A ; V, d) ; \\
-I_{C}^{(2 U)}(-A ;-V, d) & =S_{C}^{(2 L)}(A ; V, d) ; \\
-I_{C}^{(3 U)}(-A ;-V, d) & =S_{C}^{(3 L)}(A ; V, d) ; \\
-I_{C}^{(4)}(-A ;-V, d) & =S_{C}^{(4)}(A ; V, d) .
\end{aligned}
$$

Proof. The conclusion follows immediately from Proposition 2.2.

Lemma 2.1. Let $V, W \in \mathscr{P}(Y), d \in C$ and $\alpha \in \mathbb{R}$. Then

$$
W \cap\left(\bigcap_{v \in V}(v-C)+\alpha d\right)=\emptyset \Longrightarrow \alpha \leq I_{C}^{(1)}(A ; V, d), \forall A \in \mathscr{P}(Y) \text { with } A \cap W \neq \emptyset \text {. }
$$

Proof. We assume that $\alpha>I_{C}^{(1)}(A ; V, d)$ for some $A \in \mathscr{P}(Y)$ with $A \cap W \neq \emptyset$. By Proposition 2.3, we obtain

$$
A \subset\left(\bigcap_{v \in V}(v-C)+\alpha d\right)
$$


Since $A \cap W \neq \emptyset$, we have

$$
W \cap\left(\bigcap_{v \in V}(v-C)+\alpha d\right) \neq \emptyset .
$$

This is a contradiction, and therefore the conclusion follows.

Lemma 2.2. Let $V, W \in \mathscr{P}(Y), d \in C$ and $\alpha \in \mathbb{R}$. Then

$$
W \cap((V-C)+\alpha d)=\emptyset \Longrightarrow \alpha \leq I_{C}^{(3 U)}(A ; V, d), \forall A \in \mathscr{P}(Y) \text { with } A \cap W \neq \emptyset .
$$

Proof. The proof is similar to that of Lemma 2.1 .

Lemma 2.3. Let $V, W \in \mathscr{P}(Y), d \in C$ and $\alpha \in \mathbb{R}$. Then

$$
W \subset\left(\bigcap_{v \in V}(v-C)+\alpha d\right) \Longrightarrow \alpha \geq I_{C}^{(2 L)}(A ; V, d), \forall A \in \mathscr{P}(Y) \text { with } A \cap W \neq \emptyset .
$$

Proof. The proof is similar to that of Lemma 2.1.

Lemma 2.4. Let $V, W \in \mathscr{P}(Y), d \in C$ and $\alpha \in \mathbb{R}$. Then

$$
W \subset((V-C)+\alpha d) \Longrightarrow \alpha \geq I_{C}^{(4)}(A ; V, d), \forall A \in \mathscr{P}(Y) \text { with } A \cap W \neq \emptyset \text {. }
$$

Proof. The proof is similar to that of Lemma 2.1.

Lemma 2.5. Let $V, W, Q \in \mathscr{P}(Y)$ and $d \in C$. Then,

$$
W \subset Q \Longrightarrow I_{C}^{(j)}(W ; V, d) \leq I_{C}^{(j)}(Q ; V, d)
$$

for each $j=1,2 U, 3 U$.

Proof. When $j=1$, we assume that there exists $t^{\prime} \in \mathbb{R}$ such that

$$
I_{C}^{(1)}(W ; V, d)>t^{\prime}>I_{C}^{(1)}(Q ; V, d) .
$$

By Proposition 2.3, we have

$$
W \not \subset \bigcap_{v \in V}(v-C)+t^{\prime} d
$$

and

$$
Q \subset \bigcap_{v \in V}(v-C)+t^{\prime} d
$$

This contradicts $W \subset Q$. Hence,

$$
I_{C}^{(1)}(W ; V, d) \leq I_{C}^{(1)}(Q ; V, d) .
$$

When $j=2 U, 3 U$, both proofs are similar to the case of $j=1$.

Lemma 2.6. Let $V, W, Q \in \mathscr{P}(Y)$ and $d \in C$. Then,

$$
W \subset Q \Longrightarrow I_{C}^{(j)}(W ; V, d) \geq I_{C}^{(j)}(Q ; V, d)
$$

for each $j=2 L, 3 L, 4$.

Proof. The proofs are similar to those of Lemma 2.5. 


\section{INHERITATION OF SEMICONTINUITY OF SET-VALUED MAPS VIA SCALARIZATION}

We consider set-valued maps $F: X \rightarrow \mathscr{P}(Y)$, where $X$ is a topological space and we introduce a new concept of invariant property for those maps with respect to a binary relationship on a family of sets in $Y$, which is regarded as some kind of continuity from the view point of ordermonotonicity. Let $\mathscr{N}(x)$ and $\preccurlyeq$ be a neighborhood system of a point $x \in X$ and a binary relation on $\mathscr{P}(Y)$, respectively.

Definition 3.1. We define the following two binary relations on $\mathscr{P}(Y)$.

(1) $W \preccurlyeq{ }_{1} A \stackrel{\text { def }}{\Longleftrightarrow}$ int $W \cap A \neq \emptyset$,

(2) $W \preccurlyeq 2 A \stackrel{\text { def }}{\Longleftrightarrow} A \subset$ int $W$.

Definition 3.2. Let $F: X \rightarrow \mathscr{P}(Y)$ be a set-valued map, $x_{0} \in X$ and $\preccurlyeq$ a binary relation on $\mathscr{P}(Y)$. We say that $F$ is $\preccurlyeq$-continuous at $x_{0}$ if, $\forall W \subset Y$ with $W \preccurlyeq F\left(x_{0}\right)$, there exists $V \in$ $\mathscr{N}\left(x_{0}\right)$ such that $W \preccurlyeq F(x), \forall x \in V$.

For $\preccurlyeq_{1}$ and $\preccurlyeq_{2}$ as special cases, we can easily check that the corresponding notions coincide with usual "lower (semi)continuity" and "upper (semi)continuity" for set-valued maps, respectively.

(1) $F$ is lower continuous ( $\preccurlyeq 1$-continuous) at $x_{0}$ if

$$
\forall W \subset Y \text { with } W \preccurlyeq{ }_{1} F\left(x_{0}\right), \exists V \in \mathscr{N}\left(x_{0}\right) \text { s.t. } W \preccurlyeq{ }_{1} F(x), \forall x \in V .
$$

(2) $F$ is upper continuous ( $\preccurlyeq 2$-continuous) at $x_{0}$ if

$$
\forall W \subset Y \text { with } W \preccurlyeq 2 F\left(x_{0}\right), \exists V \in \mathscr{N}\left(x_{0}\right) \text { s.t. } W \preccurlyeq{ }_{2} F(x), \forall x \in V .
$$

Definition 3.3. Let $\varphi: \mathscr{P}(Y) \rightarrow \mathbb{R} \cup\{ \pm \infty\}$ be a scalarization function, $A_{0} \in \mathscr{P}(Y)$, and $\preccurlyeq$ a binary relation on $\mathscr{P}(Y)$. Then,

(1) we say that $\varphi$ is $\preccurlyeq$-lower semicontinuous at $A_{0}$ if

$$
\forall r<\varphi\left(A_{0}\right), \exists W \in \mathscr{P}(Y) \text { such that } W \preccurlyeq A_{0} \text { and } r<\varphi(A), \forall A \in U(W, \preccurlyeq) \text {, }
$$

(2) we say that $\varphi$ is $\preccurlyeq$-upper semicontinuous at $A_{0}$ if

$$
\forall r>\varphi\left(A_{0}\right), \exists W \in \mathscr{P}(Y) \text { such that } W \preccurlyeq A_{0} \text { and } r>\varphi(A), \forall A \in U(W, \preccurlyeq) \text {, }
$$

where $U(W, \preccurlyeq):=\{A \in \mathscr{P}(Y) \mid W \preccurlyeq A\}$.

Theorem 3.1. Let $F: X \rightarrow \mathscr{P}(Y), \varphi: \mathscr{P}(Y) \rightarrow \mathbb{R} \cup\{ \pm \infty\}, x_{0} \in X$, and $\preccurlyeq$ a binary relation on $\mathscr{P}(Y)$. If $F$ is $\preccurlyeq$-continuous at $x_{0}$ and $\varphi$ is $\preccurlyeq$-lower semicontinuous at $F\left(x_{0}\right)$, then $\varphi \circ F$ is lower semicontinuous at $x_{0}$ where $\varphi \circ F(x):=\varphi(F(x))$ for each $x \in X$.

Proof. For any $r<\varphi\left(F\left(x_{0}\right)\right)$, since $\varphi$ is $\preccurlyeq$-lower semicontinuous at $F\left(x_{0}\right)$, there exists $W_{0} \in$ $\mathscr{P}(Y)$ such that $W_{0} \preccurlyeq F\left(x_{0}\right)$ and $r<\varphi(A)$ for any $A \in U\left(W_{0}, \preccurlyeq\right)$. Then, since $F$ is $\preccurlyeq$-continuous at $x_{0}$, there exists $V \in \mathscr{N}\left(x_{0}\right)$ such that $W_{0} \preccurlyeq F(x)$ for any $x \in V$. It is easy to check that $F(x) \in U\left(W_{0}, \preccurlyeq\right)$ and thus we obtain $r<\varphi(F(x))$ for any $x \in V$. This means that $\varphi \circ F$ is lower semicontinuous at $x_{0}$.

Theorem 3.2. Let $F: X \rightarrow \mathscr{P}(Y), \varphi: \mathscr{P}(Y) \rightarrow \mathbb{R} \cup\{ \pm \infty\}, x_{0} \in X$, and $\preccurlyeq$ a binary relation on $\mathscr{P}(Y)$. If $F$ is $\preccurlyeq$-continuous at $x_{0}$ and $\varphi$ is $\preccurlyeq$-upper semicontinuous at $F\left(x_{0}\right)$, then $\varphi \circ F$ is upper semicontinuous at $x_{0}$. 
Proof. The proof is similar to that of Theorem 3.1.

Proposition 3.1. Let $A_{0}, V \in \mathscr{P}(Y)$ and $d \in \operatorname{int} C$. The following statements hold:

(1) $I_{C}^{(j)}(\cdot ; V, d)$ is $\preccurlyeq 1_{1}$-lower semicontinuous at $A_{0}$ for $j=1,3 U$;

(2) $I_{C}^{(j)}(\cdot ; V, d)$ is $\preccurlyeq_{1}$-upper semicontinuous at $A_{0}$ for $j=2 L, 4$.

Proof. First, we prove (i). Let $r<I_{C}^{(j)}\left(A_{0} ; V, d\right)$. It is sufficient to show that there exists $W \in$ $\mathscr{P}(Y)$ such that

$$
W \preccurlyeq 1 A_{0}
$$

and

$$
r<I_{C}^{(j)}(A ; V, d), \forall A \in U(W, \preccurlyeq 1) .
$$

Take $\alpha, \beta \in \mathbb{R}$ satisfying $r<\beta<\alpha<I_{C}^{(j)}\left(A_{0} ; V, d\right)$.

When $j=1$, we have

$$
A_{0} \not \subset \bigcap_{v \in V}(v-C)+\alpha d
$$

and

$$
\bigcap_{v \in V}(v-C)+\alpha d \supset \mathrm{cl}\left(\bigcap_{v \in V}(v-C)+\beta d\right) .
$$

It follows that there exists $y \in Y$ such that $y \in\left(\operatorname{cl}\left(\bigcap_{v \in V}(v-C)+\beta d\right)\right)^{\mathrm{c}} \cap A_{0}$. Thus we can take $W \in \mathscr{P}(Y)$ with

$$
y \in \operatorname{int} W
$$

and

$$
W \cap\left(\bigcap_{v \in V}(v-C)+\beta d\right)=\emptyset .
$$

From (3.3), $W$ satisfies (3.1). By Lemma 2.1 and (3.4), $\beta \leq I_{C}^{(1)}\left(A_{0} ; V, d\right)$ for all $A \in \mathscr{P}(Y)$ with $A \cap W \neq \emptyset$. This implies that $W$ satisfies (3.2). Therefore $I_{C}^{(1)}(\cdot ; V, d)$ is $\preccurlyeq{ }_{1}$-lower semicontinuous at $A_{0}$. When $j=3 U$, we can similarly prove the statement by using Lemma 2.2 instead of Lemma 2.1.

Next, we prove (ii). Let $r>I_{C}^{(j)}\left(A_{0} ; V, d\right)$. It is sufficient to show that there exists $W \in \mathscr{P}(Y)$ such that

$$
W \preccurlyeq{ }_{1} A_{0}
$$

and

$$
r>I_{C}^{(j)}(A ; V, d), \forall A \in U(W, \preccurlyeq 1) .
$$

Take $\alpha, \beta \in \mathbb{R}$ satisfying $r>\beta>\alpha>I_{C}^{(j)}\left(A_{0} ; V, d\right)$. When $j=2 L$, we have

$$
A_{0} \cap\left(\bigcap_{v \in V}(v-C)+\alpha d\right) \neq \emptyset
$$

and

$$
\bigcap_{v \in V}(v-C)+\alpha d \subset \operatorname{int}\left(\bigcap_{v \in V}(v-C)+\beta d\right) .
$$


It follows that there exists $y \in Y$ such that $y \in A_{0} \cap \operatorname{cl}\left(\bigcap_{v \in V}(v-C)+\beta d\right)$. Thus we can take $W \in \mathscr{P}(Y)$ with

$$
y \in \operatorname{int} W
$$

and

$$
W \subset \bigcap_{v \in V}(v-C)+\beta d .
$$

From (3.7), we have that $W$ satisfies (3.5). By Lemma 2.3 and (3.8), $\beta \geq I_{C}^{(2 L)}\left(A_{0} ; V, d\right)$ for all $A \in \mathscr{P}(Y)$ with $A \cap W \neq \emptyset$. This implies that $W$ satisfies (3.6), and therefore $I_{C}^{(2 L)}(\cdot ; V, d)$ is $\preccurlyeq 1$-lower semicontinuous at $A_{0}$. When $j=4$, we can similarly prove the statement by using Lemma 2.4 instead of Lemma 2.3.

Proposition 3.2. Let $A_{0}, V \in \mathscr{P}(Y)$ and $d \in \operatorname{int} C$. The following statements hold:

(1) $S_{C}^{(j)}(\cdot ; V, d)$ is $\preccurlyeq 1_{1}$-lower semicontinuous at $A_{0}$ for $j=2 U, 4$;

(2) $S_{C}^{(j)}(\cdot ; V, d)$ is $\preccurlyeq 1$-upper semicontinuous at $A_{0}$ for $j=1,3 L$.

Proof. The conclusion follows immediately from Propositions 2.4 and 3.1.

Proposition 3.3. Let $A_{0}, V \in \mathscr{P}(Y)$ and $d \in \operatorname{int} C$. The following statements hold:

(1) $I_{C}^{(j)}(\cdot ; V, d)$ is $\preccurlyeq 2$-upper semicontinuous at $A_{0}$ for $j=1,3 U$;

(2) $I_{C}^{(j)}(\cdot ; V, d)$ is $\preccurlyeq 2$-lower semicontinuous at $A_{0}$ for $j=2 L, 4$.

Proof. First, we prove (i). Let $r>I_{C}^{(j)}\left(A_{0} ; V, d\right)$. It is sufficient to show that there exists $W \in$ $\mathscr{P}(Y)$ such that

$$
W \preccurlyeq 2 A_{0}
$$

and

$$
r>I_{C}^{(j)}(A ; V, d), \forall A \in U(W, \preccurlyeq 2) .
$$

Take $\alpha, \beta \in \mathbb{R}$ satisfying $r>\beta>\alpha>I_{C}^{(j)}\left(A_{0} ; V, d\right)$. When $j=1$, we have

$$
A_{0} \subset \bigcap_{v \in V}(v-C)+\alpha d \subset \operatorname{int}\left(\bigcap_{v \in V}(v-C)+\beta d\right) .
$$

Putting $W:=\bigcap_{v \in V}(v-C)+\beta d$, we obtain $A_{0} \subset \operatorname{int} W$, that is, (3.9). It holds that $I_{C}^{(1)}(W ; V, d) \leq$ $\beta<r$ because $W \leq_{C}^{(1)} V+\beta d$. For any $A \in U(W, \preccurlyeq 2)$, by $A \subset W$ and Lemma $2.5, I_{C}^{(1)}(A ; V, d) \leq$ $I_{C}^{(1)}(W ; V, d)$ and thus $I_{C}^{(1)}(A ; V, d)<r$. Hence (3.10) holds, and therefore $I_{C}^{(1)}(\cdot ; V, d)$ is $\preccurlyeq 2$-upper semicontinuous at $A_{0}$. When $j=3 U$, we can similarly prove the statement.

Next we prove (ii). Let $r<I_{C}^{(j)}\left(A_{0} ; V, d\right)$. It is sufficient to show that there exists $W \in \mathscr{P}(Y)$ such that

$$
W \preccurlyeq 2 A_{0}
$$

and

$$
r<I_{C}^{(j)}(A ; V, d), \forall A \in U(W, \preccurlyeq 2) .
$$

Take $\alpha, \beta \in \mathbb{R}$ satisfying $r<\beta<\alpha<I_{C}^{(j)}\left(A_{0} ; V, d\right)$. When $j=2 L$, we have

$$
A_{0} \subset\left(\bigcap_{v \in V}(v-C)+\alpha d\right)^{\mathrm{c}} \subset \operatorname{int}\left(\bigcap_{v \in V}(v-C)+\beta d\right)^{\mathrm{c}} \text {. }
$$


Putting $W:=\left(\bigcap_{v \in V}(v-C)+\beta d\right)^{\mathrm{c}}$, we obtain $A_{0} \subset$ int $W$, that is, (3.11). It holds that $I_{C}^{(2 L)}(W ; V, d) \geq$ $\beta>r$ because $W \not_{C}^{(2 L)} V+\beta d$. For any $A \in U(W, \preccurlyeq 2)$, by $A \subset W$ and Lemma 2.6, we have

$$
I_{C}^{(2 L)}(A ; V, d) \geq I_{C}^{(2 L)}(W ; V, d) .
$$

Thus, $I_{C}^{(2 L)}(A ; V, d)>r$. Hence (3.12) holds. It follows that $I_{C}^{(2 L)}(\cdot ; V, d)$ is $\preccurlyeq 2$-lower semicontinuous at $A_{0}$. When $j=4$, we can similarly prove the statement.

Proposition 3.4. Let $A_{0}, V \in \mathscr{P}(Y)$ and $d \in \operatorname{int} C$. The following statements hold:

(1) $S_{C}^{(j)}(\cdot ; V, d)$ is $\preccurlyeq 2$-upper semicontinuous at $A_{0}$ for $j=2 U, 4$;

(2) $S_{C}^{(j)}(\cdot ; V, d)$ is $\preccurlyeq 2$-lower semicontinuous at $A_{0}$ for $j=1,3 L$.

Proof. The conclusion follows immediately from Propositions 2.4 and 3.3.

From Propositions 3.1, 3.2, 3.3, and 3.4, we have the following results as corollaries of Theorems 3.1 and 3.2; see $[11,12]$.

Theorem 3.3. Let $F: X \rightarrow \mathscr{P}(Y)$. For each $V \in \mathscr{P}(Y)$ and $d \in \operatorname{int} C$, the following statements hold:

(1) For each $j=1,3 U$,

(a) if $F$ is $\preccurlyeq_{1}$-continuous (l.s.c.) at $x_{0}$, then $I_{C}^{(j)}(F(\cdot) ; V, d)$ is lower semicontinuous at $x_{0}$,

(b) if $F$ is $\preccurlyeq 2$-continuous (u.s.c.) at $x_{0}$, then $I_{C}^{(j)}(F(\cdot) ; V, d)$ is upper semicontinuous at $x_{0}$;

(2) For each $j=2 L, 4$,

(c) if $F$ is $\preccurlyeq_{1}$-continuous (l.s.c.) at $x_{0}$, then $I_{C}^{(j)}(F(\cdot) ; V, d)$ is upper semicontinuous at $x_{0}$,

(d) if $F$ is $\preccurlyeq 2$-continuous (u.s.c.) at $x_{0}$, then $I_{C}^{(j)}(F(\cdot) ; V, d)$ is lower semicontinuous at $x_{0}$.

Theorem 3.4. Let $F: X \rightarrow \mathscr{P}(Y)$. For each $V \in \mathscr{P}(Y)$ and $d \in$ int $C$, the following statements hold:

(1) For each $j=2 U, 4$,

(a) if $F$ is $\preccurlyeq_{1}$-continuous (l.s.c.) at $x_{0}$, then $S_{C}^{(j)}(F(\cdot) ; V, d)$ is lower semicontinuous at $x_{0}$,

(b) if $F$ is $\preccurlyeq 2$-continuous (u.s.c.) at $x_{0}$, then $S_{C}^{(j)}(F(\cdot) ; V, d)$ is upper semicontinuous at $x_{0}$;

(2) For each $j=1,3 L$,

(c) if $F$ is $\preccurlyeq 1_{1}$-continuous (l.s.c.) at $x_{0}$, then $S_{C}^{(j)}(F(\cdot) ; V, d)$ is upper semicontinuous at $x_{0}$,

(d) if $F$ is $\preccurlyeq 2$-continuous (u.s.c.) at $x_{0}$, then $S_{C}^{(j)}(F(\cdot) ; V, d)$ is lower semicontinuous at $x_{0}$.

\section{Conclusion}

In the paper, we find out mathematical background and mechanism for the inheritation of the semicontinuity of set-valued maps by using general scalarization for sets, which are regarded 
as the framework of generalizations of results in earlier study. Since the unified scalarization functions for sets introduced in [10] satisfy certain desired semicontinuity, our main results can be reduced to results in $[11,12]$ as special cases.

\section{Acknowledgments}

The authors would like to dedicate the paper to Professor Christiane Tammer for her valuable contributions into nonlinear scalarization functionals. Also the authors are grateful to Professor Akhtar A. Khan as an editor-in-chief of the journal for his useful suggestions and encouragement on this research.

\section{REFERENCES}

[1] C. Gerstewitz (C. Tammer), Nichtkonvexe dualität in der vektoroptimierung, Wiss. Z. Tech. Hochsch. LeunaMerseburg 25 (1983), 357-364.

[2] A. Göpfert, H. Riahi, C. Tammer, C. Zălinescu, Variational Methods in Partially Ordered Space, Springer, New York, 2003.

[3] C. Gutiérrez, B. Jiménez, E. Miglierina, E. Molho, Scalarization in set optimization with solid and nonsolid ordering cones, J. Global Optim. 61 (2015), 525-552.

[4] A. H. Hamel, A. Löhne, Minimal element theorems and Ekeland's principle with set relations, J. Nonlinear Convex Anal. 7 (2006), 19-37.

[5] K. Ike, Y. Ogata, T. Tanaka, H. Yu, Sublinear-like scalarization scheme for sets and its applications to setvalued inequalities, In: A.A. Khan, E. Köbis, C. Tammer (eds.), Variational Analysis and Set Optimization: Developments and Applications in Decision Making, pp. 72-91, CRC Press, Boca Raton, FL, 2019

[6] K. Ike, T. Tanaka, Convex-cone-based comparisons of and difference evaluations for fuzzy sets, Optimization, 67 (2018), 1051-1066.

[7] A. A. Khan, C. Tammer, C. Zălinescu, Set-valued Optimization, Springer, Berlin, 2015.

[8] E. Köbis, M. A. Köbis, Treatment of set order relations by means of a nonlinear scalarization functional: a full characterization, Optimization 65 (2016), 1805-1827.

[9] D. Kuroiwa, T. Tanaka, T. X. D. Ha, On cone convexity of set-valued maps, Nonlinear Anal. 30 (1997), 1487-1496.

[10] I. Kuwano, T. Tanaka, S. Yamada, Characterization of nonlinear scalarizing functions for set-valued maps, In: S. Akashi, W. Takahashi, T. Tanaka (eds.), Proceedings of the Asian Conference on Nonlinear Analysis and Optimization, pp. 193-204, Yokohama Publishers, Yokohama, 2009.

[11] I. Kuwano, T. Tanaka, S. Yamada, Inherited properties of nonlinear scalarizing functions for set-valued maps, In: Edited by S.Akashi, Y.Kimura, T.Tanaka (eds.), Proceedings of the Sixth International Conference on Nonlinear Analysis and Convex Analysis, pp. 161-177, Yokohama Publishers, Yokohama, 2009.

[12] I. Kuwano, T. Tanaka, S. Yamada, Unified scalarization for sets and set-valued Ky Fan minimax inequality, J. Nonlinear Convex Anal. 11 (2010), 513-525.

[13] S. Nishizawa, T. Tanaka, P. G. Georgiev, On inherited properties of set-valued maps, Proceedings of the Third International Conference on Nonlinear Analysis and Convex Analysis, Yokohama Publishers, Yokohama, 2003, 341-350.

[14] Y. Ogata, T. Tanaka, Y. Saito, G. M. Lee, J. H. Lee, An alternative theorem for set-valued maps via set relations and its application to robustness of feasible sets, Optimization 67 (2018), 1067-1075.

[15] Y. Saito, T. Tanaka, S. Yamada, On generalization of Ricceri's theorem for Fan-Takahashi minimax inequality into set-valued maps via scalarization, J. Nonlinear Convex Anal. 16 (2015), 513-525.

[16] Y. Sonda, I. Kuwano, T. Tanaka, Cone-semicontinuity of set-valued maps by analogy with real-valued semicontinity, Nihonki Math. J. 21 (2010), 91-103. 